\title{
An Optimized Pentaplex PCR for Detecting DNA Mismatch Repair-Deficient Colorectal Cancers
}

\author{
Ajay Goel ${ }^{1 * 9}$, Takeshi Nagasaka $^{19}$, Richard Hamelin ${ }^{2}$, C. Richard Boland ${ }^{1 *}$ \\ 1 Division of Gastroenterology, Department of Internal Medicine, Baylor University Medical Center and Charles A. Sammons Cancer Center, Dallas, Texas, United States of \\ America, 2 INSERM, U762, Paris, France
}

\begin{abstract}
Purpose: Microsatellite instability (MSI) is used to screen colorectal cancers (CRC) for Lynch Syndrome, and to predict outcome and response to treatment. The current technique for measuring MSI requires DNA from normal and neoplastic tissues, and fails to identify tumors with specific DNA mismatch repair (MMR) defects. We tested a panel of five quasimonomorphic mononucleotide repeat markers amplified in a single multiplex PCR reaction (pentaplex PCR) to detect MSI.

Experimental Design: We investigated a cohort of 213 CRC patients, comprised of 114 MMR-deficient and 99 MMRproficient tumors. Immunohistochemical (IHC) analysis evaluated the expression of MLH1, MSH2, PMS2 and MSH6. MSI status was defined by differences in the quasi-monomorphic variation range (QMVR) from a pool of normal DNA samples, and measuring differences in allele lengths in tumor DNA.

Results: Amplification of 426 normal alleles allowed optimization of the QMVR at each marker, and eliminated the requirement for matched reference DNA to define MSI in each sample. Using $\geq 2 / 5$ unstable markers as the criteria for MSI resulted in a sensitivity of $95.6 \%(95 \% \mathrm{Cl}=90.1-98.1 \%)$ and a positive predictive value of $100 \%(95 \% \mathrm{Cl}=96.6 \%-100 \%)$. Detection of MSH6-deficiency was limited using all techniques. Data analysis with a three-marker panel (BAT26, NR21 and NR27) was comparable in sensitivity (97.4\%) and positive predictive value (96.5\%) to the five marker panel. Both approaches were superior to the standard approach to measuring MSI.
\end{abstract}

Conclusions: An optimized pentaplex (or triplex) PCR offers a facile, robust, very inexpensive, highly sensitive, and specific assay for the identification of MSI in CRC.

Citation: Goel A, Nagasaka T, Hamelin R, Boland CR (2010) An Optimized Pentaplex PCR for Detecting DNA Mismatch Repair-Deficient Colorectal Cancers. PLoS ONE 5(2): e9393. doi:10.1371/journal.pone.0009393

Editor: Joseph Najbauer, City of Hope National Medical Center, United States of America

Received November 3, 2009; Accepted February 3, 2010; Published February 24, 2010

Copyright: (C) $2010 \mathrm{Goel}$ et al. This is an open-access article distributed under the terms of the Creative Commons Attribution License, which permits unrestricted use, distribution, and reproduction in any medium, provided the original author and source are credited.

Funding: The present work was supported by grants RO1 CA 72851 (to CRB) and RO1 CA 129286 (to AG and CRB) from the National Cancer Institute, National Institutes of Health, and funds from the Baylor Research Institute. The funders had no role in study design, data collection and analysis, decision to publish, or preparation of the manuscript.

Competing Interests: The authors have declared that no competing interests exist.

*E-mail: ajayg@baylorhealth.edu (AG); rickbo@baylorhealth.edu (CRB)

9 These authors contributed equally to this work.

\section{Introduction}

Microsatellite instability (MSI), which is defined as the accumulation of insertion-deletion mutations at short repetitive DNA sequences (or 'microsatellites') is a characteristic feature of cancer cells with DNA mismatch repair (MMR) deficiency [1]. Inactivation of any of several MMR genes, including MLH1, MSH2, MSH6 and PMS2, can result in MSI. Originally, MSI was shown to correlate with germline defects in MMR genes in patients with Lynch syndrome (LS), where $>90 \%$ of colorectal cancer (CRG) patients exhibit MSI [2,3]. It was later recognized that MSI also occurs in $\sim 12 \%$ of sporadic CRCs occurring in patients that lack germline MMR mutations, and MSI in these patients is due to promoter methylation-induced silencing of the MLH1 gene expression [4]. Determination of MSI status in CRC has clinical use for identifying patients with germline defects predisposing to MMR-deficiency. Additionally, MSI status has prognostic and therapeutic implications, because MSI CRGs typically have a better prognosis, and these cancers are less responsive to 5FU-based adjuvant chemotherapy [5].
Since its initial discovery more than a decade ago, the methods and criteria to determine MSI in CRC have constantly evolved. However, there is still a lack of consensus on the use of various MSI assays that are more robust, inexpensive and would result in MSI analyses that best represents MMR-deficiency in laboratories worldwide [6]. In an effort to unify MSI analysis in CRC, in 1997 an National Cancer Institute (NCI) workshop recommended using a reference panel of five MSI markers that consisted of 2 mononucleotide repeat markers (BAT26 and BAT25) and 3 dinucleotide repeat markers (D2S123, D5S346 and D17S250) [7]. In a follow-up NCI workshop, the panel recognized some of the limitations of the original markers, primarily due to the inclusion of the 3 dinucleotide markers [8]. First, it was recognized that the dinucleotide repeat markers were more suitable for identifying MSI-L tumors, while mononucleotide repeat markers were more specific and sensitive for the determination of MSI (or MSI-H) CRCs [9]. Second, due to the polymorphic nature of dinucleotide markers, these required the availability of not just tumor but matching normal DNA from each individual to interpret MSI results. It has been shown that a panel of five quasi-monomorphic 
mononucleotide repeat markers in a pentaplex PCR obviate the need for normal DNA from each CRC patient, and may offer better specificity and sensitivity than the NCI-panel markers [10].

Unfortunately, in spite of its obvious strengths, the pentaplex MSI approach has gained limited acceptance for MSI-based screening of CRCs. There may be several reasons for this, including a lack of clear understanding on the technical aspects and independent validation of this assay. This study addresses this concern by validating the accuracy of the pentaplex-panel markers in a large series of MMR-proficient and deficient GRCs by analyzing PCR-amplified profiles of each marker in both tumor and matching normal DNA. Herein, we demonstrate a highly sensitive and specific pentaplex PCR assay that requires one-time optimization of quasi-monomorphic variation range (QMVR) for each marker in normal DNA. We provide evidence that an optimized pentaplex PCR assay should be the preferred method for MSI evaluation in clinical and research laboratories, as it is rapid, economical, highly sensitive and specific for detecting MMR-deficient CRGs and obviates the need for reference normal DNA.

\section{Materials and Methods}

\section{Ethics Statement}

All patients provided written informed consent and the study was approved by institutional review boards of Baylor University Medical Center, Dallas, USA; University of Heidelberg, Heidelberg, Germany; and the Okayama University Hospital, Okayama, Japan.

\section{Tissue Specimens}

Tumor and matching germline DNA was collected from 213 patients diagnosed with CRC at three different institutions: 1) Baylor University Medical Center, Dallas, TX, USA 2) University of Heidelberg, Heidelberg, Germany and 3) Okayama University Hospital, Okayama, Japan. Among this cohort, 114 tumors were MMR-deficient, and included 50 CRGs with loss of MLH1, 48 with loss of MSH2 and 8 cases each with the exclusive loss of PMS2 or MSH6 proteins. The remaining 99 cases were MMRproficient.

\section{MMR Protein Immunohistochemistry}

We examined protein expression for MLH1, MSH2, PMS2, and MSH6 in 213 tumor tissues by immunohistochemical (IHC) staining using DAKO EnVision System-HRP polymer system kit (Dako Cytomation Inc., Carpinteria, CA). Tissue sections were probed with appropriate dilutions of mouse monoclonal antibodies against MLH1 (clone 13271A, BD Pharmingen, San Diego, CA), MSH2 (clone FE11, Oncogene Research Products, Boston, MA), PMS2 (clone A37, BD Pharmingen San Diego, CA), and MSH6 protein (clone 44, BD Transduction Laboratories, Lexington, KY). Tumor cells were scored negative for MMR protein expression only if the epithelial cells within the tumor tissue lacked nuclear staining, while the surrounding stromal cells still showed positive staining.

\section{Microdissection and DNA Amplification}

Serial sections $(5 \mu \mathrm{m})$ from formalin-fixed paraffin-embedded matched normal and tumor tissues were routinely stained, and representative normal and tumor regions were identified by microscopic examination. Genomic DNA was isolated from the paraffin-embedded tissues using the QIAamp DNA mini kit (Qiagen, Valencia, CA) following separation of tumor and normal tissue by manual microdissection.

\section{Pentaplex PCR and Quasi-Monomorphic Variation Range} (QMVR) Definition

MSI analysis was carried out using five mononucleotide repeat microsatellite targets (BAT-25, BAT-26, NR-21, NR-24 and NR27) in a pentaplex PCR system [10]. Primer sequences have been described previously, and each sense primer was end-labeled with one of the fluorescent markers: FAM, HEX or NED [11]. Pentaplex PCR was performed in an MJ Research DNA 200 multicycler (Biorad, Hercules, CA). The PCR conditions consisted of an initial $15 \mathrm{~min}$ denaturation step at $95^{\circ} \mathrm{C}$, followed by 35 cycles at $95^{\circ} \mathrm{C}$ for $30 \mathrm{~s}, 55^{\circ} \mathrm{C}$ for $30 \mathrm{~s}$ and $72^{\circ} \mathrm{C}$ for $30 \mathrm{~s}$, with a final extension at $72^{\circ} \mathrm{C}$ for $10 \mathrm{~min}$. Amplified PCR products were diluted with formamide, and run on an Applied Biosystems 3100 Avant automated capillary electrophoresis DNA sequencer. Allelic sizes for each of the markers were estimated using GeneMapper 3.1 software (Applied Biosystems, Foster City, CA).

For the determination and validation of the quasi-monomorphic variation range (QMVR) for each of the five MSI markers, PCR amplification profiles were scored individually, and the size of both alleles was determined for each marker and for each tumor individually as described previously [11]. For calculation purposes, due to the monomorphic nature of these markers, we calculated each allele size twice in homozygous samples.

\section{Determination of Allelic Variations in Tumor DNA Compared to Normal DNA}

Next, we investigated whether the availability of matching normal DNA from a CRC patient would enhance the screening performance of the pentaplex PCR in MMR deficient CRGs. We calculated the differences in allelic lengths between tumor and normal DNA for each patient and each MSI marker. In each case, we regarded the shortest allele present in tumor or normal DNA for calculation purposes using the following formula:

(Difference in allele length) $=\mid$ (Normal DNA allele)-(Tumor DNA allele) | (bp)

If the PCR fragment from tumor DNA revealed two peaks, we considered the shorter peak representative of tumor DNA.

\section{MSI Analysis by NCI-Panel Markers}

To compare the sensitivity and specificity of pentaplex PCR with the original NCI-panel of markers (BAT25, BAT26, D2S123, D5S346 and D17S250), we performed MSI analyses on a subset of 86 MMR-deficient CRGs and 37 MMR proficient CRGs using both approaches [7]. Primers for each of the 5 markers were previously described $[12,13]$.

\section{Statistical Analyses}

We used logistic regression analysis to examine the diagnostic performance for MMR deficient CRCs utilizing different strategies to define MSI. To examine the relationship between individual MSI markers, a multivariate correlation and hierarchical clustering analysis was performed using standardized absolute difference length between tumor allele and the germline/normal allele. Analyses were performed using JMP (version 6.0, SAS Institute). All reported P values are twosided and $\mathrm{P}<0.05$ was considered statistically significant.

\section{Results}

\section{MMR-Deficient and MMR-Proficient CRCs}

To determine the accuracy of a pentaplex PCR system for detecting MMR deficient CRCs, we investigated a cohort of 213 CRCs which comprised of 114 MMR-deficient and 99 MMRproficient tumors. Table 1 lists the clinical features of MMRproficient and -deficient CRGs. 
Table 1. Characteristics of CRC patients according to MMR protein expression status.

\begin{tabular}{llll}
\hline & & & \\
\hline MMR expression status & Mean Age (95\%Cl) & Male, $\boldsymbol{n}$ (\%) & Amsterdam Criteria II Positive, $\boldsymbol{n}$ (\%) \\
\hline MLH1 Deficient $(\mathrm{n}=50)$ & $44.0(40.0-48.1)$ & $35(70)$ & $46(92)$ \\
MSH2 Deficient $(\mathrm{n}=48)$ & $43.3(39.9-46.7)$ & $32(68)$ & $45(94)$ \\
PMS2 Deficient $(\mathrm{n}=8)$ & $44.0(29.0-60.0)$ & $4(50)$ & $0(0)$ \\
MSH6 Deficient $(\mathrm{n}=8)$ & $33.3(27.5-39.0)$ & $3(38)$ & $0(0)$ \\
MMR Proficient $(\mathrm{n}=99)$ & $47.2(43.8-50.7)$ & $49(49)$ & $0(0)$ \\
\hline
\end{tabular}

doi:10.1371/journal.pone.0009393.t001

\section{Determination and Optimization of QMVR for Each Marker by Normal DNA}

Although the five mono-repeat markers in pentaplex PCR have been suggested to be highly monomorphic in germline DNA from a wide spectrum of populations worldwide, the success of this MSI assay heavily relies on the accurate determination of QMVR for each marker in normal DNA [11]. Theoretically the QMVR for each marker should be constant in each experimental setting, but data indicates that specific instrumentation or reagents may partly influence allele size measurements for each marker [11]. This mandates one-time careful determination of QMVR in germline DNA prior to tumor MSI analysis. We PCR amplified 426 alleles from 213 normal DNA specimens to determine the QMVR for each MSI marker. As shown in Figure 1, the polymorphic range for each MSI marker in normal DNA is as follows: NR 27 (8287 bp), NR21 (102-106 bp), NR24 (120-125 bp), BAT25 (142$148 \mathrm{bp})$, and BAT26 (174-179 bp). The most common allele for each of the markers was as follows: NR27 (85 bp), NR21 (105 bp), NR24 (123 bp), BAT25 (145 bp) and BAT26 (178 bp). In contrast to previous studies which used healthy subjects to generate QMVR for each marker [11], QMVR values in our study were based on a large series of matching normal DNA samples obtained from CRG patients. We noticed that our most common alleles were shorter for each marker (BAT26 by 1 bp, NR21 \& NR27 by
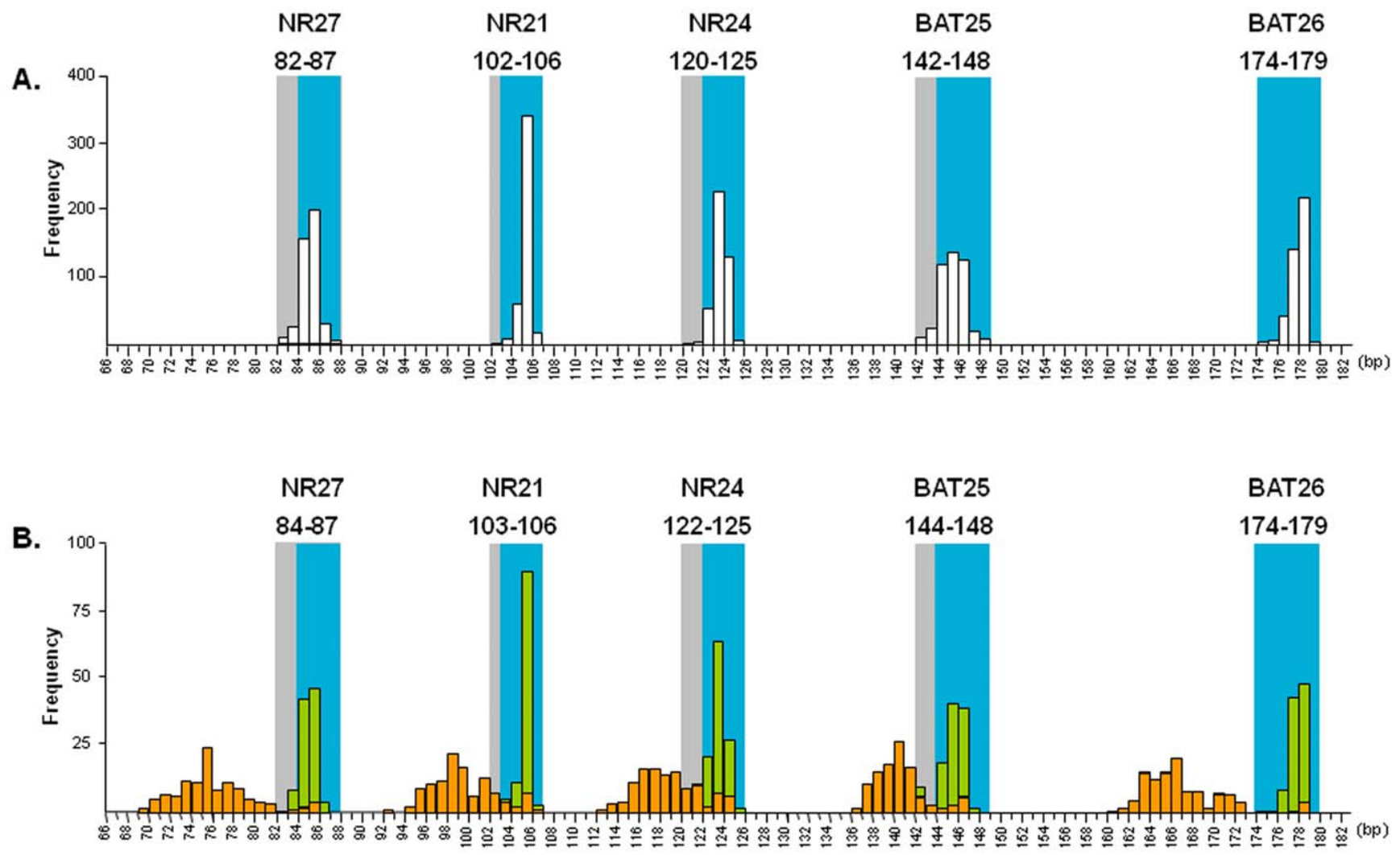

Normal DNA

MMR Proficient

MMR Deficient

Figure 1. Frequency of allele size distribution for the five pentaplex markers. A) Allele size distribution (in base pairs) from 213 normal DNA specimens. For each marker, blue shading indicates the adjusted QMVR, while the gray shading indicates the entire range of allelic size obtained from 426 germline alleles. B) Distribution of allele sizes in MMR-deficient (orange) and MMR-proficient (green) CRCs. doi:10.1371/journal.pone.0009393.g001 
2 bp and BAT25 \& NR24 by 3 bp) and therefore optimized QMVR were shifted slightly leftwards as indicated by grey shaded areas in Figure 1A. Thereafter, we considered a tumor to be positive for allelic variation (or unstable) at a given marker when tumor allele sizes did not fall within the optimized QMVR. Supporting the specificity of our newly optimized QMVR, we noted that almost all amplification profiles from all MMRproficient tumors fell within this range, while almost all MMRdeficient tumors showed large allelic variations at multiple markers and were outside of this range (Figure 1B).

\section{Determination of Allelic Variations in Tumor DNA for} Each Marker and Its Relationship to MMR Status in CRC

We noted that due to significant concordance between MMR IHC and MSI results at each marker, we utilize IHC information for the calculation of cut-off thresholds that corresponded with loss of DNA MMR protein expression and MMR-deficiency. We defined cut-offs for each of the five markers by determining specific allele length variations in which at least 95\% of tumors were within QMVR (showed no instability) and were simultaneously MMR-proficient according to IHC results. In this manner, we successfully determined that an allelic difference of $>3$ bp between the tumor and corresponding normal DNA for both BAT25 and BAT26 was diagnostic for a MSI-positive tumor. Similarly, a difference of $>2$ bp in tumor versus germline DNA for NR21, NR24, and NR27 was considered positive for defining MSI-positivity (gray shaded square box in Figure S1).

\section{Performance Characteristics of Individual Pentaplex Markers for the Identification of MMR-Deficient CRCs}

We next examined the performance characteristics of individual pentaplex markers, particularly the relatively understudied NRmarkers for identifying MMR-deficient CRCs (Table 2). Our analyses using 'QMVR values alone' clearly highlighted the robustness of various mono-markers to detect MMR-deficient CRCs with a sensitivity that varied from $86.8 \%$ to $94.7 \%$, and a specificity of $96.0 \%$ to $100 \%$, for identifying MMR proficient CRCs. Of interest, when results were re-analyzed using 'data from the matching normal DNA', the results were strikingly similar, wherein each marker displayed a sensitivity range of $85.1 \%$ to 95.6\% for identifying MMR-deficient CRCs, and a specificity of $95.0 \%$ to $100 \%$ for detecting MMR-proficient CRCs.
An Optimized Pentaplex PCR Does Not Require Matching Normal DNA to Detect MMR-Deficient CRC

We next analyzed the performance of all mono-markers in the pentaplex PCR assay in both MMR deficient and -proficient CRCs using two approaches: first, using 'QMVR results alone' (Figure 2A left panels and Table 3), and second, when data were available from the 'matching normal DNA from CRC patients' (Figure 2A, right panels and Table 4). When allelic variations at $\geq 3$ of 5 markers was defined as diagnosis of MSI, both strategies displayed $93.9 \%$ (CI, 87.9\%-97.0\%) sensitivity for identification of MMR-deficient CRCs and 100\% (CI, 96.3\%-100\%) specificity for MMR-proficient CRCs. With respect to correlation of MSI data with MMR protein expression status, both strategies demonstrated similar sensitivity for tumors with MLH1 (96.0\%), MSH2 (100\%), and PMS2 (100\%) deficiency. However, neither approach was sufficiently robust to detect MSH6-deficiency and identified only 37.5\% (3/8) of MSH6-deficient CRCs (Figure 2B). Contrarily, when MSI-H was defined by instability at $\geq 2$ of 5 markers, both strategies demonstrated slightly improved sensitivity for MMR-deficient CRCs (95.6\%; CI, 90.1\%-98.1\%) and same specificity for MMR-proficient CRCs (100\%; CI, 96.3\%-100\%). But this improvement was only due to increased sensitivity for detecting MSH6-deficient tumors (62.5\%; 5 of 8 CRCs), without any associated change in sensitivity for identification of MLH1 (96.0\%), MSH2 (100\%), and PMS2 (100\%) deficient (Figure 2B). Hence, the optimized pentaplex assay is highly specific and sensitive for detecting MMR-deficient CRCs, and that the availability of normal DNA from a CRC patient does not necessarily enhance its performance. Additionally, using a cut-off for instability at $\geq 2 / 5$ markers to define MSI results in maximal sensitivity and specificity for this assay, particularly for samples mutant for MSH6.

\section{Association between Pentaplex Mononucleotide Repeat Markers}

We then asked whether an association exists among individual mononucleotide repeat markers, or whether the instability at each marker was an independent event. For this, we performed multivariate correlation as well as hierarchical clustering analysis by comparing the differences in allelic sizes obtained from tumor and normal DNA (Figures 3A\&B$)$. All correlation coefficients $(r)$ demonstrated values of over 0.75 , suggesting a strong mutual

Table 2. Performance characteristics of each MSI Marker by different strategies for the identification of MMR-Deficient CRCs.

\begin{tabular}{|c|c|c|c|c|c|}
\hline Marker & Reference & Sensitivity \% $(95 \% \mathrm{Cl})$ & Specificity \% $(95 \% \mathrm{Cl})$ & PPV \% $(95 \% \mathrm{Cl})^{\#}$ & NPV $\%(95 \% \mathrm{Cl})^{+}$ \\
\hline \multirow[t]{2}{*}{ BAT25 } & QMVR & $90.4(83.5-94.5)$ & $96.0(90.1-98.4)$ & $96.2(90.8-98.5)$ & $89.6(82.4-94.1)$ \\
\hline & Normal DNA & $86.0(78.4-91.2)$ & $97.0(91.5-99.0)$ & $97.0(91.6-99.0)$ & 85.7 (78.0-91.0) \\
\hline \multirow[t]{2}{*}{ BAT26 } & QMVR & 94.7 (89.0-97.6) & $97.0(91.5-99.0)$ & $97.3(92.4-99.1)$ & $94.1(87.8-97.3)$ \\
\hline & Normal DNA & $95.6(90.1-98.1)$ & $97.0(91.5-99.0)$ & $97.3(92.4-99.1)$ & 95.1 (88.9-97.9) \\
\hline \multirow[t]{2}{*}{ NR21 } & QMVR & 87.7 (80.4-92.5) & $100(96.3-100)$ & $100(96.3-100)$ & $87.6(80.3-92.5)$ \\
\hline & Normal DNA & $89.5(82.5-93.9)$ & $100(96.3-100)$ & $100(96.4-100)$ & $89.2(82.0-93.7)$ \\
\hline \multirow[t]{2}{*}{ NR24 } & QMVR & 86.8 (79.4-91.9) & $99.0(94.5-99.8)$ & $99.0(94.6-99.8)$ & 86.7 (79.2-91.8) \\
\hline & Normal DNA & $85.1(77.4-90.5)$ & $95.0(88.7-97.8)$ & $95.1(89.0-97.9)$ & $84.7(76.8-90.2)$ \\
\hline \multirow[t]{2}{*}{ NR27 } & QMVR & 93.9 (87.9-97.0) & $99.0(94.5-99.8)$ & 99.1 (94.9-99.8) & $93.3(86.9-96.7)$ \\
\hline & Normal DNA & 94.7 (98.0-97.6) & $96.0(90.1-98.4)$ & 96.4 (91.2-98.6) & 94.1 (87.6-97.2) \\
\hline
\end{tabular}

\#PPV = positive predictive value.

${ }^{+} \mathrm{NPV}=$ negative predictive value.

doi:10.1371/journal.pone.0009393.t002 

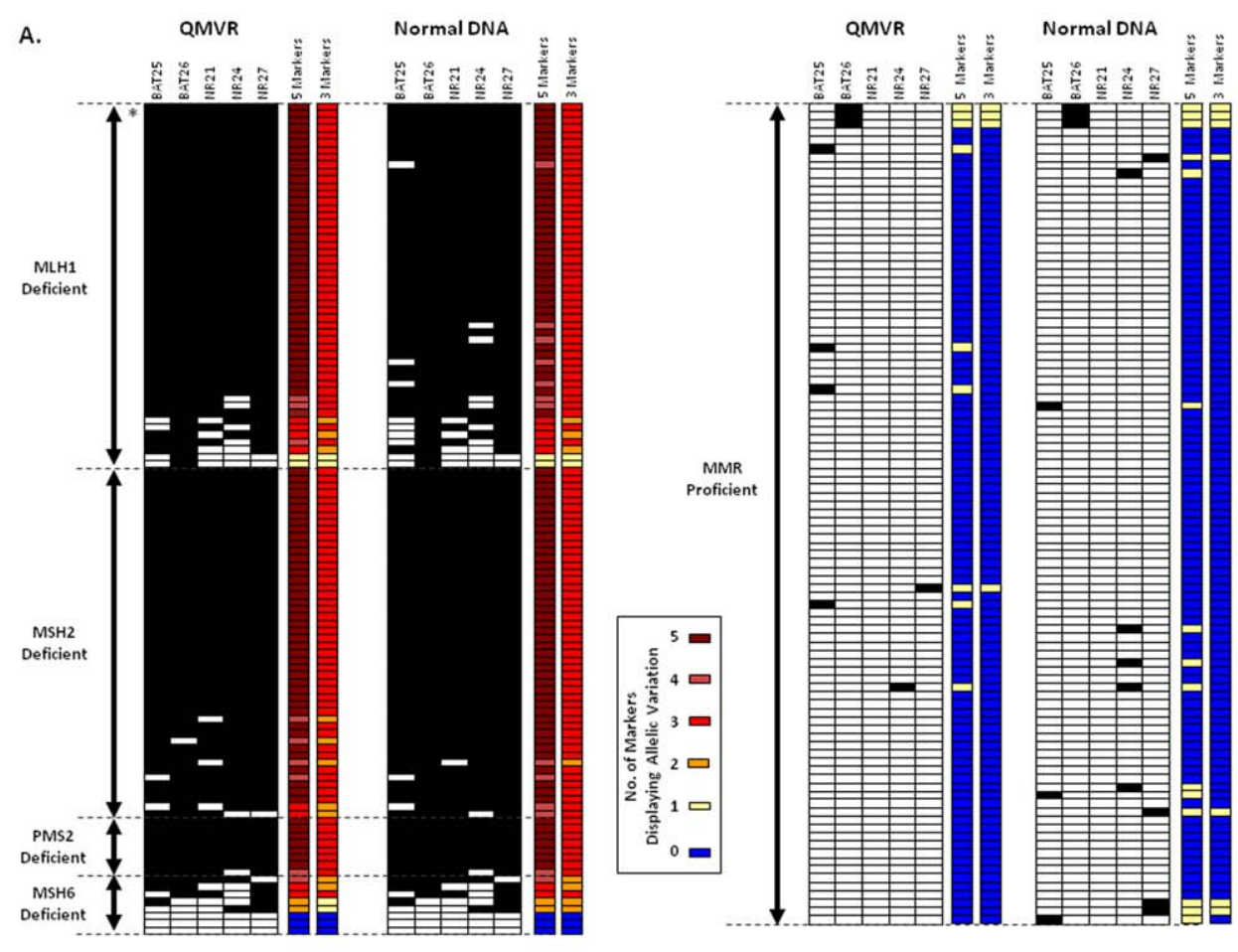

B.

口QMVR $\square$ Normal DNA

c.

口QMVR $\quad$ Normal DNA
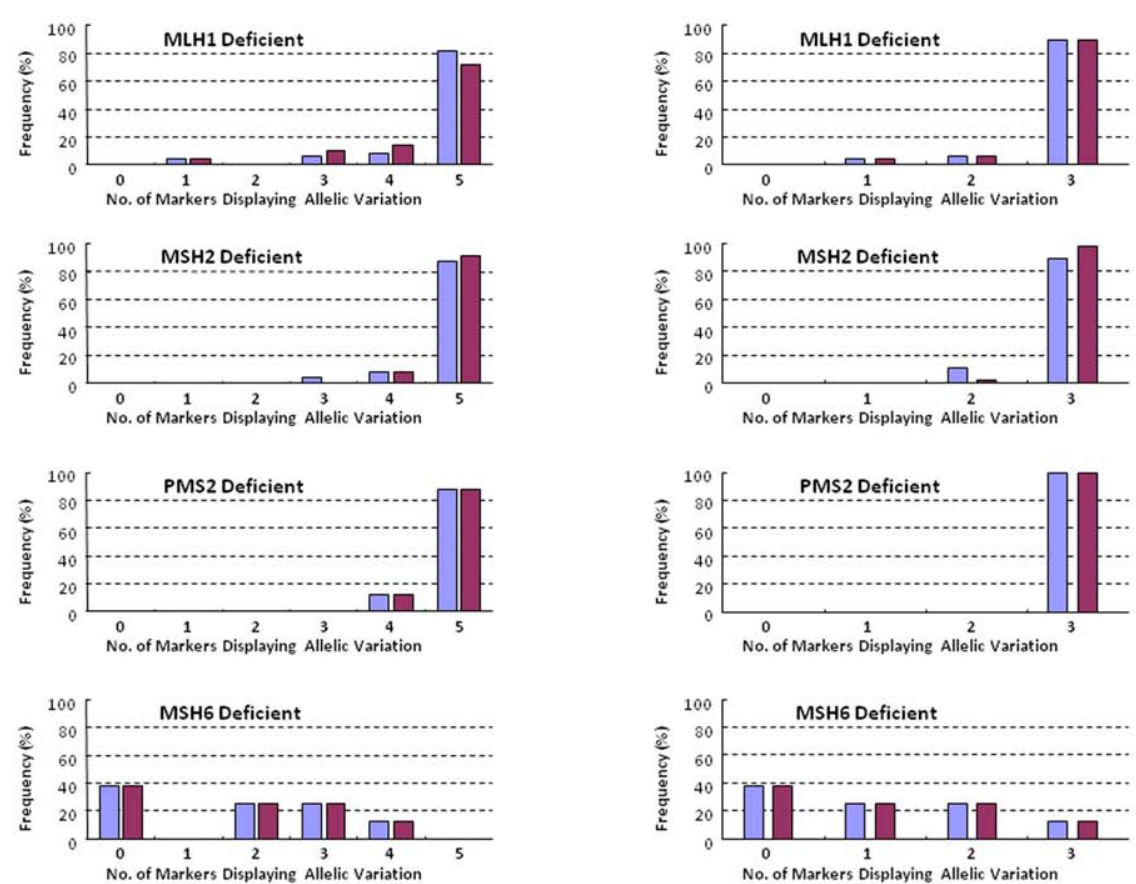

Figure 2. Performance characteristics of pentaplex markers based on QMVR, availability of normal DNA, and numbers of markers required to determine MSI in colorectal cancers. A) The figure illustrates the performance of the pentaplex mononucleotide-repeat maker panel when defining the MSI status of tumor DNA by "QMVR only" (when matched normal DNA was not available) and by "Normal DNA" by subtracting the germline allele lengths from tumor for each tumor. Data in the two panels on the left is from MMR-deficient tumors, while the other two panels on the right represent MMR-proficient CRCs. (* indicates one case with loss of both MLH1 and MSH2). Black squares indicate a tumor positive for allelic variation (i.e., unstable) and white squares indicate a tumor negative for any allele variations (i.e., stable). B) Shows the frequency of MMR-deficient tumors with number of markers displaying allelic variations when data were analyzed from all five pentaplex markers. C) Shows the frequency of MMR-deficient tumors with number of markers displaying allelic variations when data were analyzed from just three pentaplex markers (BAT26, NR21 and NR27).

doi:10.1371/journal.pone.0009393.g002 
Table 3. Performance characteristics of Pentaplex PCR system with reference to "QMVR" for Identification of MMR-deficient CRCs.

\begin{tabular}{|c|c|c|c|c|c|c|c|c|}
\hline \multirow[t]{2}{*}{$\begin{array}{l}\text { No. of Markers } \\
\text { Displaying Allelic } \\
\text { Variation }\end{array}$} & \multicolumn{4}{|c|}{ The Five Marker Panel* } & \multicolumn{4}{|c|}{ The Three Marker Panel** } \\
\hline & Sensitivity (\%) & Specificity (\%) & PPV (\%) $\#$ & NPV $(\%)^{+}$ & Sensitivity (\%) & Specificity (\%) & PPV (\%) & NPV $(\%)^{+}$ \\
\hline 5 & $78.9(70.6-85.4)$ & $100(96.3-100)$ & $100(95.9-100)$ & $80.5(72.6-86.5)$ & & & & \\
\hline 4 & $87.7(80.4-92.5)$ & $100(96.3-100)$ & $100(96.3-100)$ & $87.6(80.3-92.5)$ & & & & \\
\hline 3 & $93.9(87.9-97.0)$ & $100(96.3-100)$ & $100(96.5-100)$ & $93.4(87.0-96.8)$ & $85.1(77.4-90.5)$ & $100(96.3-100)$ & $100(96.2-100)$ & $85.3(77.8-90.6)$ \\
\hline 2 & $95.6(90.1-98.1)$ & $100(96.3-100)$ & $100(96.6-100)$ & $95.2(89.2-97.9)$ & $93.9(87.9-97.0)$ & $100(96.3-100)$ & $100(96.5-100)$ & $93.4(87.0-96.8)$ \\
\hline 1 & $97.4(92.5-99.1)$ & $90.9(83.6-95.1)$ & $92.5(86.4-96.0)$ & $96.8(90.9-98.9)$ & $97.4(92.5-99.1)$ & $96.0(90.1-98.4)$ & $96.5(91.4-98.6)$ & $96.9(91.4-99.0)$ \\
\hline
\end{tabular}

Results are expressed as percentages (\%), with 95\% confidence intervals in parentheses.

*The Five Pentaplex Marker Panel composed of BAT25, BAT26, NR21, NR24 and NR27 markers.

**The Three Pentaplex Marker Panel composed of BAT26, NR21 and NR27 markers.

\#PPV = positive predictive value.

${ }^{+} \mathrm{NPV}=$ negative predictive value.

doi:10.1371/journal.pone.0009393.t003

association among the markers. However, when analyzing specific pairwise associations, we observed that BAT26, NR21 and NR27 showed higher correlation coefficients between one another (Figure 3A). These observations were reconfirmed upon hierarchical clustering analysis, wherein we noticed that NR24 was farthest from the top of the hierarchical tree, followed by BAT25 in comparison to the BAT26, NR21 and NR27 microsatellite repeats which were more tightly correlated (Figure 3B).

\section{Reduced Marker Combination Is Equally Effective as All Five Markers in the Pentaplex Assay}

We questioned whether a reduced panel of markers might be equally effective as all five markers in the pentaplex panel. For this, we re-analyzed the screening performance of pentaplex PCR to detect MMR-deficient CRGs based upon all five, or a selected panel of three (BAT26, NR21 and NR27) markers for MSI classification analysis (Tables 3 \& $\mathbf{4}$ ).

When MSI was defined as instability at $\geq 1$ or $\geq 2$ of 3 markers, once again, comparable degrees of sensitivity $(93.9 \%-97.4 \%)$ and specificity $(92.9 \%-100 \%)$ were obtained. With respect to MMR protein expression status, both strategies displayed similar sensitivity for tumors with MLH1 (96.0\%), MSH2 (100\%), and
PMS2 (100\%) deficiency. However, as noted previously with a five marker panel, a cut-off threshold of instability at $\geq 2$ of 3 markers resulted in increased sensitivity for the detection of MSH6 deficient CRCs $(62.5 \%$; 5 of 8 tumors; Figure 2G). Although the sensitivity of an MSI assay using these criteria is marginally lower 93.9\% (CI 87.9\%-97.0\%) compared to using all five markers $95.6 \%$ (CI $90.1 \%-98.1 \%$ ), the specificity of this assay remained unchanged $(100 \%$, with both marker panels).

\section{Screening Performance of the Pentaplex Assay Is Better than with the NCl-Panel Markers}

The NCI-panel of MSI markers (2 mono markers; BAT25 \& BAT26 and 3 dinucleotide markers; D3S1023, D5S346 \& $\mathrm{D} 17 \mathrm{~S} 250)$ is currently the standard for MSI-determination in CRGs [7]. The dinucleotide markers in this panel require simultaneous amplification of matched normal DNA for the same patient with CRC, and are better suited for detecting MSI-L than MSI tumors. Since pentaplex markers are quasi-monomorphic, we compared the screening performance of these two MSI assays for the identification of the two most commonly defective MMR proteins, MLH1 and MSH2 in our collection of CRGs. As shown in Figures $4 \mathbf{A} \& \mathbf{B}$, the pentaplex markers demonstrated better or

Table 4. Performance characteristics of Pentaplex PCR system with reference to "normal DNA" for the identification of MMRdeficient CRCs.

\begin{tabular}{|c|c|c|c|c|}
\hline \multirow[t]{2}{*}{$\begin{array}{l}\text { No. of Markers } \\
\text { Displaying Allelic } \\
\text { Variation }\end{array}$} & \multicolumn{4}{|c|}{ The Five Marker Panel* } \\
\hline & Sensitivity (\%) & Specificity (\%) & PPV (\%) ${ }^{\#}$ & NPV (\%) ${ }^{+}$ \\
\hline 5 & $76.3(67.7-83.2)$ & $100(96.3-100)$ & $100(95.8-100)$ & $78.6(70.6-84.8)$ \\
\hline 4 & $87.7(80.4-92.5)$ & $100(96.3-100)$ & $100(96.3-100)$ & $87.6(80.3-92.5)$ \\
\hline 3 & 93.9 (87.9-97.0) & $100(96.3-100)$ & $100(96.5-100)$ & $93.4(87.0-96.8)$ \\
\hline 2 & $95.6(90.1-98.1)$ & $100(96.3-100)$ & $100(96.6-100)$ & $95.2(89.2-97.9)$ \\
\hline 1 & $97.4(92.5-99.1)$ & $84.9(76.5-90.6)$ & $88.1(81.3-92.7)$ & $96.6(90.3-98.8)$ \\
\hline \multicolumn{5}{|c|}{ 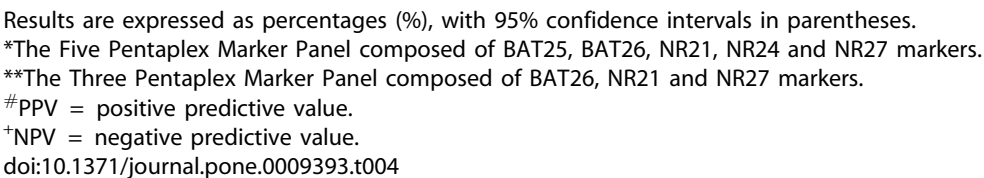 } \\
\hline
\end{tabular}

The Three Marker Panel**

Sensitivity (\%) Specificity (\%) PPV (\%) ${ }^{\#} \quad$ NPV (\%) ${ }^{+}$

Sensitivity (\%) Specificity (\%) PPV (\%) $\quad$ NPV (\%)

$\begin{array}{llll}88.6(87.5-93.2) & 100(96.3-100) & 100(96.3-100) & 88.4(81.1-93.1) \\ 93.9(87.9-97.0) & 100(96.3-100) & 100(96.5-100) & 93.4(87.0-96.8) \\ 97.4(92.5-99.1) & 92.9(86.1-96.5) & 94.1(88.3-97.1) & 96.8(91.1-98.9)\end{array}$


A.

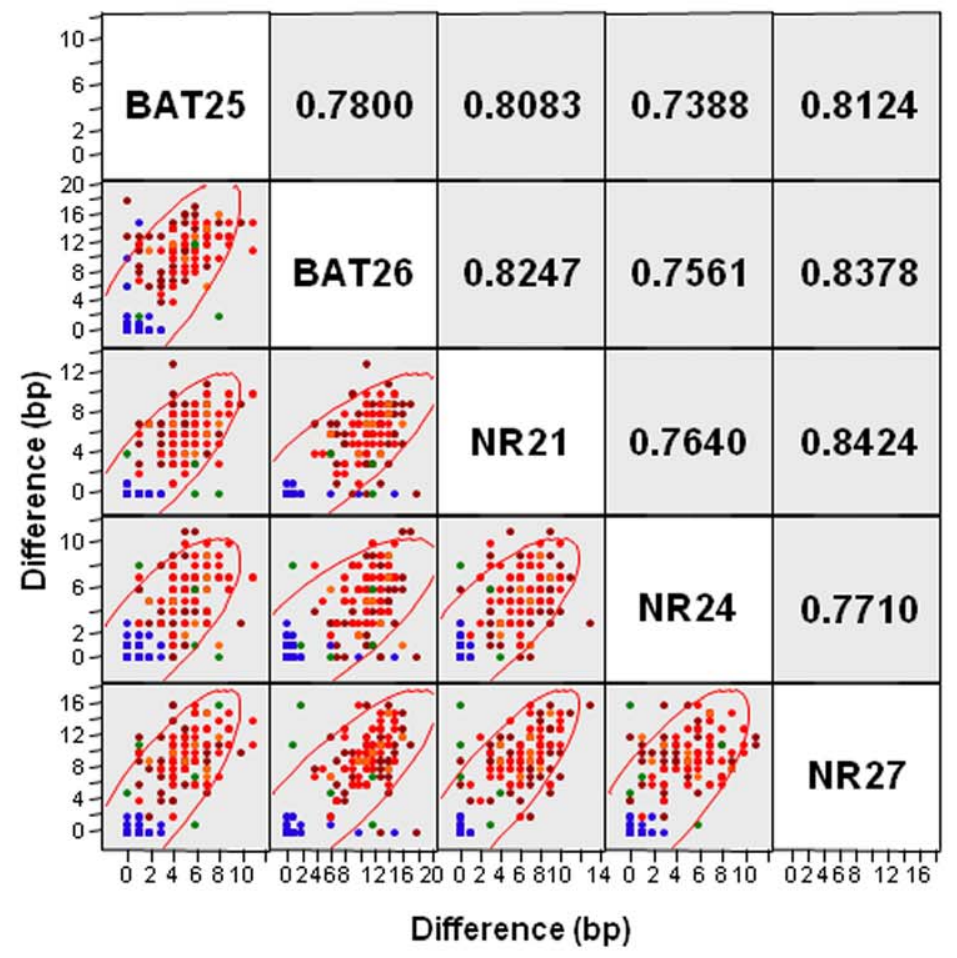

- MMR Proficient

- MLH1 Deficient

- MSH2 Deficient

O PMS2 Deficient

MSH6 Deficient
B.

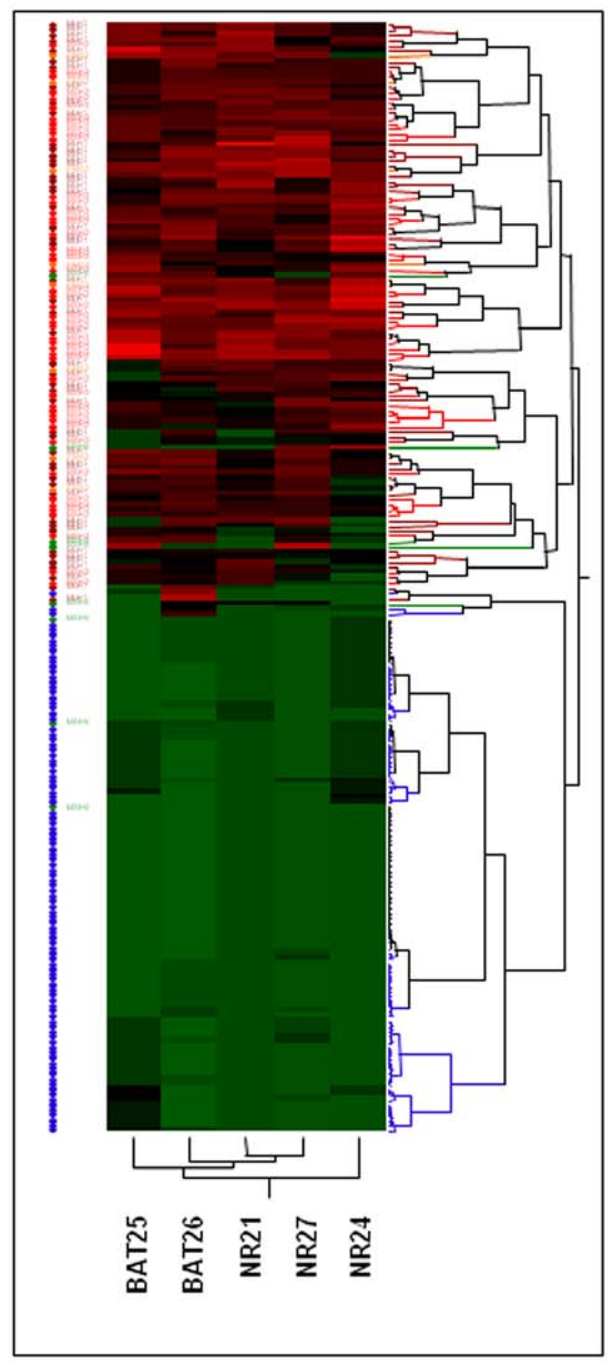

Figure 3. Correlation between various mono-nucleotide markers in the pentaplex PCR. A) A scatter-plot matrix demonstrating the pairwise correlation coefficient $(r)$ between five microsatellite markers in the cohort of MMR-proficient and deficient CRCs. The $Y$ and $X$-axis denote absolute differences in allele sizes between the tumor DNA and normal DNA. B) The figure shows hierarchical clustering analysis derived from 104 MMR-deficient and 99 MMR-proficient CRCs. The data are presented in matrix format in which the rows represent each CRC and the columns indicate the individual mononucleotide markers. The color scale represents the gradient (green to red) of absolute allele length differences between tumor and germline DNA from QMVR standardized data; green (no differences in allele size between tumor and normal DNA) to red (significant differences in allelic lengths between tumor and normal DNA).

doi:10.1371/journal.pone.0009393.g003

comparable sensitivity and specificity to the NCI panel markers for the identification of MMR-deficient CRCs. Given this scenario, the pentaplex PCR offers tremendous overall advantage over NCI-markers, as it is more rapid, utilizes a single PCR reaction, obviates the need for normal DNA, is less expensive and is highly accurate.

\section{Discussion}

The goal of this study was to develop a rapid and highly accurate MSI assay that can be adapted in any laboratory equipped with an automated DNA sequencer. Herein, we optimized and validated the usefulness of five mononucleotide microsatellite markers that can be amplified in a single pentaplex PGR reaction for MSI determination in a large series of MMRproficient and deficient CRGs.
MSI analysis with the NCI-panel of five microsatellite markers (2 mono- and 3 di-nucleotide repeats) still appears to be a preferred method in most clinical and research laboratories. Unfortunately, although multiple studies have repeatedly shown that mononucleotide MSI markers offer higher accuracy for detecting MSI-H or MMR-deficient tumors [10], this approach has not gained sufficient recognition or acceptance. One of the key technical challenges of this assayis the need for careful one-time optimization of QMVR for each mono-marker. This is because allelic size estimation for these quasi-monomorphic markers can be influenced by the use of specific reagents or the sequencing machine [14]. Supporting this concept, the QMVR for all markers in our patient population differed by a few base pairs than what had been reported previously $[11,14]$. We believe that the QMVRs in our study are more robust, as these were obtained from the matching normal/germline DNA from a large series of 
A.

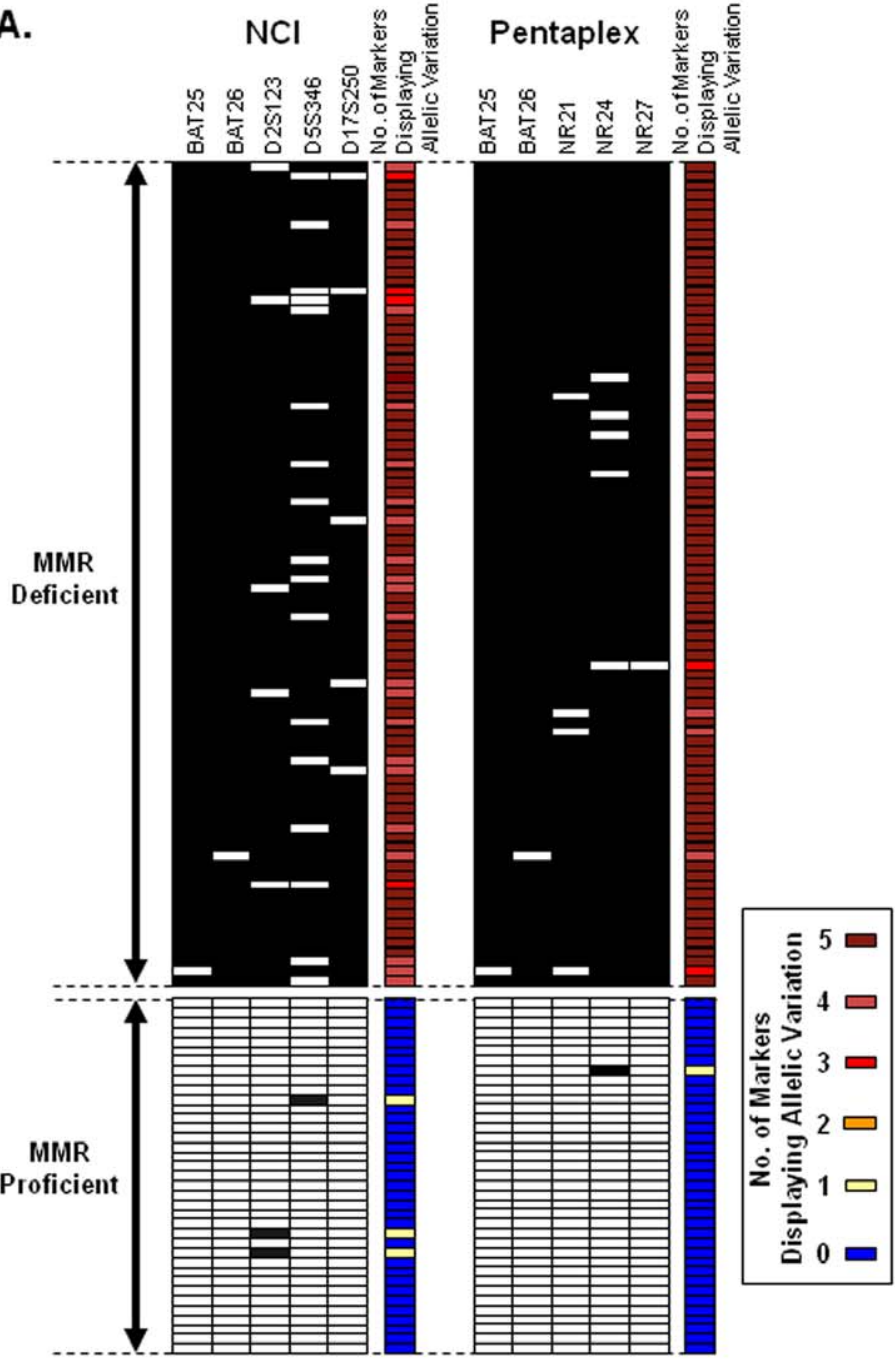

B.
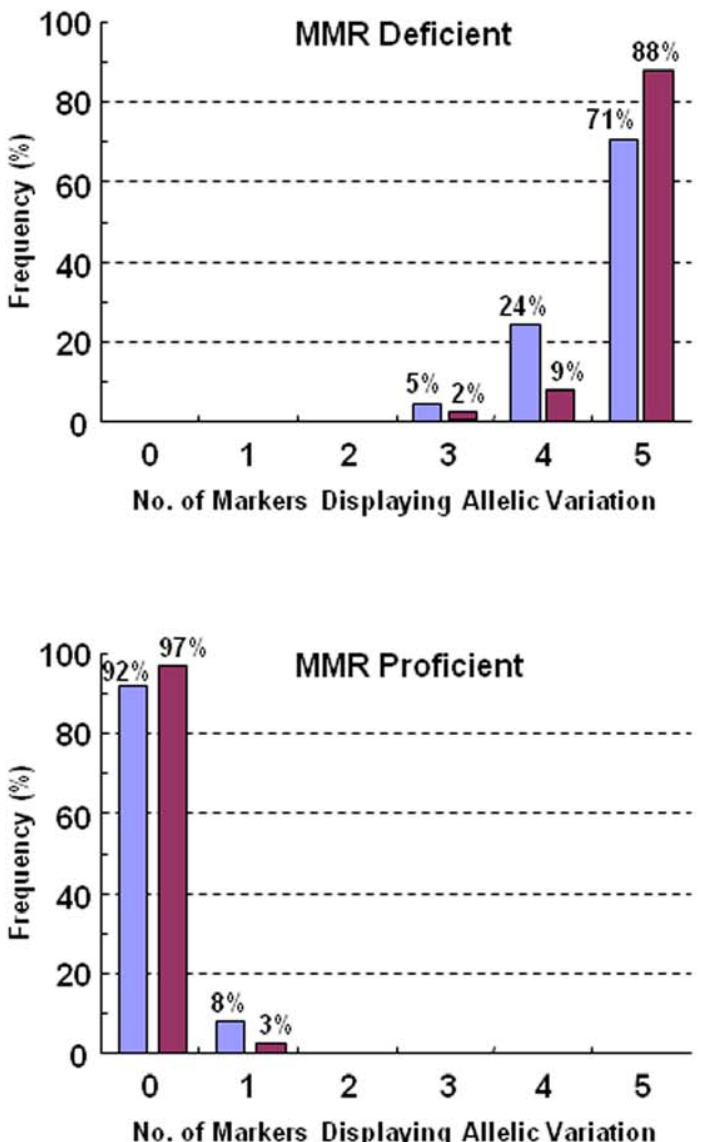

$\square \mathrm{NCl} \quad \square$ Pentaplex

Figure 4. Comparison between pentaplex PCR and NCI panel of markers to determine MMR-deficiency in colorectal cancers. A) The figure shows the performance comparison between the $\mathrm{NCl}$ panel markers and the QMVR optimized pentaplex PCR. Black squares indicate a tumor positive for allelic variation (or unstable) and white squares indicate a tumor negative for any allele variations (or stable). The dinucleotide repeat markers (D2S123, D5S346 and D17S250) are less robust than the mononucleotide repeats for detecting MSI. B) The figure illustrates the frequency of tumors with number of markers displaying allelic variations in MMR-deficient and proficient CRCs. As indicated, pentaplex PCR shows a higher sensitivity and specificity compared to $\mathrm{NCl}$ panel of markers, and the distribution of altered markers is unambiguously bimodal.

doi:10.1371/journal.pone.0009393.g004

CRC patients, instead from healthy individuals as reported previously $[11,14]$. Previous studies have highlighted the usefulness of BAT25 and BAT26 markers to identify MSI-positive CRCs [9], however, there is limited understanding on the sensitivities and positive predictive values of the NR-markers. A recent report indicated that a screen based on an assessment of only BAT26 and NR24 may be effective for the detection of MMR-deficient CRGs [15]. In fact, BAT26 had the highest sensitivity and positive predictive value in our cohort of MMR-deficient CRCs. On the contrary, pairwise correlation and hierarchical clustering analysis in our study clearly showed weakest predictive values for NR24 (and BAT25), compared to the remaining three markers (BAT26, NR21, and NR27). BAT26 is a quasi-monomorphic marker which is located immediately 3' to $\mathrm{MSH} 2$ exon 5 , and is considered to be very sensitive and specific for MSI testing. However, large deletions in $\mathrm{MSH} 2$ that include the BAT26 locus are not uncommon in CRC, and in such instances, although the tumor is MMR-deficient, PCR at the BAT26 locus will result in the false negative amplification of the wild-type alleles from the normal cells in the tumor mass [16]. These data caution against the conventional wisdom that although BAT26 is frequently used for MSI-determination, using BAT26 alone, or in conjunction with a less accurate marker such as NR24 can underestimate MSI, and will preclude detection of potential MMR-deficient CRCs. In addition, our observation of the high sensitivity and positive predictive value for a reduced panel of three markers (BAT26, NR21 and NR27) versus all five makers have economic implications for future MSI-based assays.

Another critical issue with the use of the pentaplex assay is lack of agreement on the minimum number of unstable markers required to classify a tumor as MSI. In this context, the original report suggested $\geq 3 / 5$ unstable markers in tumor DNA would define a MSI-positive CRC [10], while a subsequent study suggested that instability at only $\geq 2 / 5$ markers was sufficient to detect a MMR-deficient CRG [15]. We revisited this issue by analyzing data through multiple approaches and our results 
demonstrate that the sensitivity and specificity of the pentaplex PCR was unaffected regardless of whether we considered a cut-off of $\geq 2$ or $\geq 3$ of 5 markers for the detection of MLH1, MSH2 and PMS2-deficient CRCs. However, we propose that a criterion of $\geq 2$ of 5 unstable markers is more accurate, as it enhanced the screening performance of the assay by identifying additional MSH6-deficient tumors without adding any false positives.

One of the limitations of the NCI-panel of markers is its inability to identify MSH6-deficient CRCs. Our data indicate that the use of mono-markers in the pentaplex panel can identify the majority of MSH6 deficient GRGs. This is of significance because the MutS $\alpha$ complex, a heterodimer of MSH2 and MSH6, preferentially recognizes base/base mismatches as well as small insertion/deletion loops containing 1 or 2 unpaired nucleotides in the DNA sequence and directs the repair of these lesions [17]. Therefore, one would expect that the functional loss of MutS $\alpha$ due to MSH6-deficiency would lead to preferential instability in the loci containing mononucleotide repeats [18].

In conclusion, we present evidence that favor the use of an optimized pentaplex PCR system to screen for MMR-deficient CRCs. Our data indicate that a one-time optimized QMVR obviates the need for amplification of matched normal DNA to determine instability in the tumor tissue, and that instability at $\geq 2$ of 5 markers provides the most robust strategy to identify MMRdeficient CRCs. Our data suggest that a marker panel consisting of BAT26, NR21 and NR27 markers was as accurate as the fivemarker panel for MSI analysis. Importantly, the pentaplex markers showed a higher sensitivity for diagnosing MSH6deficient CRCs. We propose that this assay will replace existing

\section{References}

1. Bronner CE, Baker SM, Morrison PT, Warren G, Smith LG, et al. (1994) Mutation in the DNA mismatch repair gene homologue hMLH1 is associated with hereditary non-polyposis colon cancer. Nature 368: 258-261.

2. Ionov Y, Peinado MA, Malkhosyan S, Shibata D, Perucho M (1993) Ubiquitous somatic mutations in simple repeated sequences reveal a new mechanism for colonic carcinogenesis. Nature 363: 558-561.

3. Thibodeau SN, Bren G, Schaid D (1993) Microsatellite instability in cancer of the proximal colon. Science 260: 816-819.

4. Kane MF, Loda M, Gaida GM, Lipman J, Mishra R, et al. (1997) Methylation of the hMLH1 promoter correlates with lack of expression of hMLH1 in sporadic colon tumors and mismatch repair-defective human tumor cell lines. Cancer Res 57: 808-811.

5. Ribic CM, Sargent DJ, Moore MJ, Thibodeau SN, French AJ, et al. (2003) Tumor microsatellite-instability status as a predictor of benefit from fluorouracilbased adjuvant chemotherapy for colon cancer. N Engl J Med 349: 247-257. 10.1056/NEJMoa022289 [doi];349/3/247 [pii].

6. Laghi L, Bianchi P, Malesci A (2008) Differences and evolution of the methods for the assessment of microsatellite instability. Oncogene 27: 6313-6321.

7. Boland CR, Thibodeau SN, Hamilton SR, Sidransky D, Eshleman JR, et al. (1998) A National Cancer Institute Workshop on Microsatellite Instability for cancer detection and familial predisposition: development of international criteria for the determination of microsatellite instability in colorectal cancer. Cancer Res 58: 5248-5257.

8. Umar A, Boland CR, Terdiman JP, Syngal S, de la CA, et al. (2004) Revised Bethesda Guidelines for hereditary nonpolyposis colorectal cancer (Lynch syndrome) and microsatellite instability. J Natl Cancer Inst 96: 261-268.

9. Perucho M (1999) Correspondence re: C.R. Boland et al., A National Cancer Institute workshop on microsatellite instability for cancer detection and familial predisposition: development of international criteria for the determination of methodologies and help improve MSI-based CRC screening in the future.

\section{Supporting Information}

Figure S1 Frequency of allele size differences (in bp) between normal and tumor DNA at each marker, and the MSI status determination by QMVR (horizontal bars in blue and red on the left side) as well as by the status of MMR protein expression by IHC (horizontal bars in green and orange on the right side). The numbers on the $\mathrm{Y}$-axis represent the allele sizes difference (in bp) between normal and tumor DNA. The numbers in red reflect the microsatellite instability cut-off ranges determined for each of the markers based upon their deviation from the QMVR range and IHC data.

Found at: doi:10.1371/journal.pone.0009393.s001 (1.59 MB TIF)

\section{Acknowledgments}

We would like to thank Johannes Gebert, $\mathrm{PhD}$ and Matthias Kloor, MD (Institute of Pathology, University of Heidleberg, Germany) for generously providing the colon cancer specimens, and for their helpful discussions and critical review of the manuscript.

\section{Author Contributions}

Conceived and designed the experiments: AG TN RH. Performed the experiments: AG TN. Analyzed the data: AG TN RH. Contributed reagents/materials/analysis tools: AG RH. Wrote the paper: AG CRB.

microsatellite instability in colorectal cancer. Cancer Res., 58: 5248-5257, 1998. Cancer Res 59: 249-256.

10. Suraweera N, Duval A, Reperant M, Vaury C, Furlan D, et al. (2002) Evaluation of tumor microsatellite instability using five quasimonomorphic mononucleotide repeats and pentaplex PCR. Gastroenterology 123: 1804-1811.

11. Buhard O, Cattaneo F, Wong YF, Yim SF, Friedman E, et al. (2006) Multipopulation analysis of polymorphisms in five mononucleotide repeats used to determine the microsatellite instability status of human tumors. J Clin Oncol 24: $241-251$.

12. Goel A, Arnold CN, Niedzwiecki D, Chang DK, Ricciardiello L, et al. (2003) Characterization of sporadic colon cancer by patterns of genomic instability. Cancer Res 63: 1608-1614.

13. Goel A, Nagasaka T, Arnold GN, Inoue T, Hamilton C, et al. (2007) The CpG island methylator phenotype and chromosomal instability are inversely correlated in sporadic colorectal cancer. Gastroenterology 132: 127-138.

14. Buhard O, Suraweera N, Lectard A, Duval A, Hamelin R (2004) Quasimonomorphic mononucleotide repeats for high-level microsatellite instability analysis. Dis Markers 20: 251-257.

15. Xicola RM, Llor X, Pons E, Castells A, Alenda C, et al. (2007) Performance of different microsatellite marker panels for detection of mismatch repair-deficient colorectal tumors. J Natl Cancer Inst 99: 244-252.

16. Pastrello C, Baglioni S, Tibiletti MG, Papi L, Fornasarig M, et al. (2006) Stability of BAT26 in tumours of hereditary nonpolyposis colorectal cancer patients with MSH2 intragenic deletion. Eur J Hum Genet 14: 63-68. 5201517 [pii];10.1038/sj.ejhg.5201517 [doi]

17. Modrich $\mathrm{P}(2006)$ Mechanisms in eukaryotic mismatch repair. J Biol Chem 281: 30305-30309.

18. Verma L, Kane MF, Brassett C, Schmeits J, Evans DG, et al. (1999) Mononucleotide microsatellite instability and germline MSH6 mutation analysis in early onset colorectal cancer. J Med Genet 36: 678-682. 